PROCEEDINGS OF THE

AMERICAN MATHEMATICAL SOCIETY

Volume 134, Number 12, December 2006, Pages 3475-3483

S 0002-9939(06)08514-5

Article electronically published on June 12, 2006

\title{
RESOLUTIONS OF IDEALS OF FAT POINTS WITH SUPPORT IN A HYPERPLANE
}

\author{
GIULIANA FATABBI, BRIAN HARBOURNE, AND ANNA LORENZINI
}

(Communicated by Michael Stillman)

\begin{abstract}
Let $Z^{\prime}$ be a fat point subscheme of $\mathbb{P}^{d}$, and let $x_{0}$ be a linear form such that some power of $x_{0}$ vanishes on $Z^{\prime}$ (i.e., the support of $Z^{\prime}$ lies in the hyperplane $H$ defined by $x_{0}=0$, regarded as $\mathbb{P}^{d-1}$ ). Let $Z(i)=H \cap Z^{\prime}(i)$, where $Z^{\prime}(i)$ is the subscheme of $\mathbb{P}^{d}$ residual to $x_{0}^{i}$; note that $Z(i)$ is a fat points subscheme of $\mathbb{P}^{d-1}=H$. In this paper we give a graded free resolution of the ideal $I\left(Z^{\prime}\right)$ over $R^{\prime}=K\left[\mathbb{P}^{d}\right]$, in terms of the graded minimal free resolutions of the ideals $I(Z(i)) \subset R=K\left[\mathbb{P}^{d-1}\right]$. We also give a criterion for when the resolution is minimal, and we show that this criterion always holds if $\operatorname{char}(K)=0$.
\end{abstract}

\section{INTRODUCTION}

Let $R=K\left[\mathbb{P}^{d-1}\right]=K\left[x_{1}, \ldots, x_{d}\right]$ and $R^{\prime}=K\left[\mathbb{P}^{d}\right]=K\left[x_{0}, x_{1}, \ldots, x_{d}\right]$ be the homogeneous coordinate rings of projective space, over an algebraically closed field $K$ of arbitrary characteristic. We regard $\mathbb{P}^{d-1}$ as the hyperplane $x_{0}=0$ in $\mathbb{P}^{d}$. We will denote homogeneous components by subscripts; thus, for example, $R_{1}$ denotes the $K$-vector space of homogeneous linear forms in $K\left[\mathbb{P}^{d-1}\right]$.

Given points $p_{1}, \ldots, p_{r} \in \mathbb{P}^{d-1}$ and nonnegative integers $m_{i}$, we have the fat point subschemes $Z=m_{1} p_{1}+\cdots+m_{r} p_{r} \subset \mathbb{P}^{d-1}$ (so $I(Z) \subset R$ ) and $Z^{\prime}=m_{1} p_{1}+$ $\cdots+m_{r} p_{r} \subset \mathbb{P}^{d}$ (so $I\left(Z^{\prime}\right) \subset R^{\prime}$ ). In particular, the ideal $I(Z)$ is $I_{1}^{m_{1}} \cap \cdots \cap I_{r}^{m_{r}}$, where $I_{j}$ is the ideal generated by all forms in $R$ vanishing at $p_{j}$, and $I\left(Z^{\prime}\right)$ is $\left(I^{\prime}\right)_{1}^{m_{1}} \cap \cdots \cap\left(I^{\prime}\right)_{r}^{m_{r}}$, where $\left(I^{\prime}\right)_{j}$ is the ideal generated by all forms in $R^{\prime}$ vanishing at $p_{j}$.

We also have the obvious canonical inclusion $R \subset R^{\prime}$, so we can regard ideals in $R$ as $R$-submodules of $R^{\prime}$. Now define $Z_{m-i}^{\prime}=\left(m_{1}-i\right)_{+} p_{1}+\cdots+\left(m_{r}-i\right)_{+} p_{r} \subset \mathbb{P}^{d}$ and $Z_{m-i}=\left(m_{1}-i\right)_{+} p_{1}+\cdots+\left(m_{r}-i\right)_{+} p_{r} \subset \mathbb{P}^{d-1}$ for each $0 \leq i \leq m=$ $\max \left\{m_{1}, \ldots, m_{r}\right\}$, where for any integer $n$ we define $n_{+}=\max \{n, 0\}$. Note that $Z^{\prime}=Z_{m}^{\prime}$ and $Z=Z_{m}$, and that $\emptyset=Z_{0} \subset Z_{1} \subset \cdots \subset Z_{m}=Z$. Alternatively, let $Z^{\prime}(i)$ denote the subscheme of $\mathbb{P}^{d}$ defined by the ideal $I\left(Z^{\prime}\right):\left(x_{0}^{i}\right)$ (and thus residual to $\left.x_{0}^{i}\right)$; then $Z^{\prime}(i)=Z_{m-i}^{\prime}$ and $Z_{m-i}$ is the subscheme $Z^{\prime}(i) \cap \mathbb{P}^{d-1}$ of $\mathbb{P}^{d-1}$.

Received by the editors January 21, 2005 and, in revised form, July 7, 2005.

2000 Mathematics Subject Classification. Primary 13D02, 13D40; Secondary 14M05, 14M20.

The authors thank MURST, whose national project Algebra Commutativa e Computazionale, and the University of Perugia, whose project Metodi algebrici e analitici nello studio delle varietà supported visits to Perugia by the second author, who also thanks the NSA and NSF for supporting his research. The authors also thank the referee for helpful suggestions. 
In this paper, we construct a graded free resolution of $I\left(Z^{\prime}\right)$ over $R^{\prime}$, given graded minimal free resolutions of each $I\left(Z_{i}\right)$ over $R$. Under the condition that $I\left(Z_{i+1}\right) \subset R_{1} I\left(Z_{i}\right)$ for each $i$, we show that the constructed resolution is minimal. We also show that this condition always holds if $\operatorname{char}(K)=0$. In fact, we do not know any examples where the condition does not hold.

As a corollary we obtain a result about the Poincaré polynomial of $I\left(Z^{\prime}\right)$. (Recall that the Poincaré polynomial encodes the Betti numbers of a resolution. Given a subscheme $W \subset \mathbb{P}^{n}$ and its ideal $I(W) \subset A=K\left[\mathbb{P}^{n}\right]$, we have its minimal free resolution $0 \rightarrow F_{t} \rightarrow \cdots \rightarrow F_{1} \rightarrow F_{0} \rightarrow I(W) \rightarrow 0$. The Poincaré polynomial $P(W)$ is defined as follows. Each syzygy module $F_{i}$ is a free graded $A$-module of the form $F_{j}=\bigoplus_{i} A(-i)^{a_{i j}}$, and we take

$$
P(W)=\sum_{i j} a_{i j} T^{i} X^{j} \in \mathbf{Z}[X, T] .
$$

So, for example, the empty subscheme $W=\emptyset$, whose ideal is thus (1), has polynomial $P(W)=1$, and if $W=p$ is a single reduced point in $\mathbb{P}^{3}$, then $P(W)=$ $3 T+3 X T^{2}+X^{2} T^{3}$.)

Theorem 1.1. Let $Z^{\prime}=m_{1} p_{1}+\cdots+m_{r} p_{r} \subset \mathbb{P}^{d}$, where the points $p_{i}$ lie in a hyperplane of $\mathbb{P}^{d}$. If $Z_{i}$ is defined as above and $I\left(Z_{i+1}\right) \subset R_{1} I\left(Z_{i}\right)$ for each $i$, then

$$
P\left(Z^{\prime}\right)=(1+X T)\left(\sum_{0<i \leq m} T^{m-i} P\left(Z_{i}\right)\right)+T^{m}
$$

where $m$ is the maximum of the multiplicities $m_{1}, \ldots, m_{r}$.

We give our resolution construction in Section 2, To most usefully apply our construction, we also need examples in which the condition $I\left(Z_{i+1}\right) \subset R_{1} I\left(Z_{i}\right)$ holds. More generally, given $p_{i} \in \mathbb{P}^{d}$ and fat points $Z=m_{1}^{\prime} p_{1}+\ldots+m_{r}^{\prime} p_{r}$ and $Y=m_{1} p_{1}+\ldots+m_{r} p_{r}$ with $m_{i}^{\prime}>m_{i}$ for all $i$ whenever $m_{i}^{\prime}>0$, it seems possible that $I(Z) \subset R_{1} I(Y)$ always holds. We do not know of any counterexamples, and we show in Section 3 various situations, such as whenever $\operatorname{char}(K)=0$, where this condition does hold. We also discuss various examples in Section 3. In particular, our construction allows us to determine the resolution of the ideal of two fat points in any projective space, recovering some results (but not the explicit formulas) of FL and $[\mathrm{V}$. Since it is easy to give the resolution of any number of fat points in $\mathbb{P}^{1}$, our result also allows us, among other cases, to determine the resolution for any number of fat points in any projective space if the points lie on a line.

One can speculate on possible generalizations. In one direction, giving a resolution of an ideal $I \subset R^{\prime}$ is equivalent to giving a resolution for $R^{\prime} / I$, and in the cases we study here the annihilator of $R^{\prime} / I$ contains a power of a linear form. Perhaps our approach can be applied in other cases of modules over a polynomial ring annihilated by a power of a linear form. But even for cyclic modules it is unclear what kind of criterion to expect for resolutions to be minimal. Since our immediate interest is minimal resolutions of ideals of fat points, we have not pursued such questions here. In another direction, one can ask to relax the condition of considering points that lie only on a hyperplane. However, this would seem to change the problem in a fundamental way, since we implicitly use the fact that $R^{\prime}$ modulo a linear form is not only a quotient of $R^{\prime}$ but also a subring of $R^{\prime}$. 


\section{The COnstruction}

We will need to refer to the following two elementary results.

Lemma 2.1. Let $h: M \rightarrow N$ be a (not necessarily graded) homomorphism of graded $R$-modules. Let $F$ and $G$ be free modules over $R$ with surjective $R$-homomorphisms $\alpha: F \rightarrow M$ and $\beta: G \rightarrow N$. Then there is an $R$-homomorphism $h_{0}: F \rightarrow G$ such that $\beta h_{0}=h \alpha$, where $h_{0}$ is homogeneous of degree $t$ if $h$ is homogeneous of degree $t$. If, furthermore, $h(M) \subset R_{1} N$, then, in addition, $h_{0}$ can be chosen such that $h_{0}(F) \subset R_{1} G$.

Proof. The first part is clear, since $F$ is free, so assume $h(M) \subset R_{1} N$ and consider $B: G^{d} \rightarrow R_{1} N \subset N$, where $B:\left(c_{1}, \ldots, c_{d}\right) \mapsto x_{1} \beta\left(c_{1}\right)+\cdots+x_{d} \beta\left(c_{d}\right)$. Clearly, $B$ is surjective, so we can lift $h \alpha: F \rightarrow M \rightarrow R_{1} N$ to $h^{\prime}: F \rightarrow G^{d}$. We also have the canonical map $\gamma: G^{d} \rightarrow G$ (in which $\left.\left(c_{1}, \ldots, c_{d}\right) \mapsto x_{1} c_{1}+\cdots+x_{d} c_{d}\right)$, and $\beta \gamma=B$, and hence $\beta \gamma h^{\prime}=B h^{\prime}$. If we take $h_{0}=\gamma h^{\prime}$, then $\beta h_{0}=B h^{\prime}=h \alpha$ as desired, and $h_{0}(F) \subset \gamma\left(G^{d}\right)=R_{1} G$.

By recursively applying the previous lemma, we obtain:

Corollary 2.2. Let $h: M \rightarrow N$ be a (not necessarily graded) homomorphism of graded $R$-modules, with $F_{\bullet}$ and $G_{\bullet}$ free resolutions over $R$ of $M$ and $N$, respectively. Then there are $R$-homomorphisms $h_{j}: F_{j} \rightarrow G_{j}, j \geq 0$, compatible with the differential morphisms of the resolutions, where each $h_{j}$ is homogeneous of degree $t$ if $h$ is homogeneous of degree $t$. Moreover, if $h(M) \subset R_{1} N$, then the maps $h_{j}$ can be chosen such that $h_{j}\left(F_{j}\right) \subset R_{1} G_{j}$ for every $j$.

Now consider $\emptyset=Z_{0} \subset \cdots \subset Z_{m}=Z \subset \mathbb{P}^{d-1}$ and $Z^{\prime} \subset \mathbb{P}^{d}$ as in the introduction. Given minimal graded free resolutions (over $R$ ) for each $I\left(Z_{i}\right)$, we now construct a graded resolution (over $R^{\prime}$ ) for $I\left(Z^{\prime}\right)$. We will use the following notation: the graded free modules in the resolution of $I\left(Z_{i}\right)$ will be denoted $F_{i, j}$ (so $F_{i, 0}$ is the free $R$-module on the generators of $I\left(Z_{i}\right)$, with the suitable shifts; $F_{i, 1}$ the free $R$-module on the first syzygies of $I\left(Z_{i}\right)$, with the suitable shifts; etc.). The free generators for $F_{i, j}$ will be denoted $s_{k, i, j}$, indexed by $k$. The graded resolution differential $F_{i, j+1} \rightarrow F_{i, j}$ will be denoted $\phi_{i, j+1}$. We denote the augmentation maps $F_{i, 0} \rightarrow I\left(Z_{i}\right)$ by $\phi_{i, 0}$.

We will also need the maps $f_{i+1, j}: F_{i+1, j} \rightarrow F_{i, j}$ guaranteed by Corollary 2.2. where, in the notation of the corollary, $h: M \rightarrow N$ is the inclusion $M=I\left(Z_{i+1}\right) \subset$ $I\left(Z_{i}\right)=N$, and $f_{i+1, j}=h_{j}$. By the corollary, each $f_{i+1, j}$ is homogeneous of degree 0 and $f_{i+1, j} \phi_{i+1, j+1}=\phi_{i, j+1} f_{i+1, j+1}$, for $j \geq 0$, and $\phi_{i+1,0}=\phi_{i, 0} f_{i+1,0}$.

We now construct a resolution of $I\left(Z^{\prime}\right)$ of the form

$$
\cdots \stackrel{\phi_{j+2}^{\prime}}{\rightarrow} F_{j+1}^{\prime} \stackrel{\phi_{j+1}^{\prime}}{\rightarrow} F_{j}^{\prime} \stackrel{\phi_{j}^{\prime}}{\rightarrow} \cdots \stackrel{\phi_{1}^{\prime}}{\rightarrow} F_{0}^{\prime} \stackrel{\phi_{0}^{\prime}}{\rightarrow} I\left(Z^{\prime}\right) \rightarrow 0 .
$$

To avoid repeatedly having to indicate certain shifts explicitly, we denote $F_{i, j}(-(m-i)) \otimes_{R} R^{\prime}$ by $F_{i, j}^{\prime}$. Each map $\phi_{i, j}: F_{i, j} \rightarrow F_{i, j-1}$ induces an obvious map $F_{i, j}(-(m-i)) \rightarrow F_{i, j-1}(-(m-i))$ which extends to give a map $\phi_{i, j}^{\prime}$ : $F_{i, j}^{\prime} \rightarrow F_{i, j-1}^{\prime}$; i.e., after accounting for the shift, $\phi_{i, j}^{\prime}$ is just $\phi_{i, j} \otimes_{R} \mathrm{id}_{R^{\prime}}$. Similarly, $f_{i+1, j}^{\prime}: F_{i+1, j}^{\prime}(-1) \rightarrow F_{i, j}^{\prime}$ denotes the map coming from $f_{i+1, j}$. Now take

$$
F_{0}^{\prime}=\bigoplus_{i=0}^{m} F_{i, 0}^{\prime}
$$


and, for $j \geq 1$,

$$
F_{j}^{\prime}=F_{0, j}^{\prime} \oplus\left(\bigoplus_{i=1}^{m}\left(F_{i, j}^{\prime} \oplus F_{i, j-1}^{\prime}(-1)\right)\right)
$$

(note $F_{0, j}^{\prime}=0$ for $j \geq 1$; we include it in $F_{j}^{\prime}$ for consistency). Define the augmentation map as

$$
\phi_{0}^{\prime}\left(s_{k, i, 0} \otimes 1\right)=\phi_{i, 0}^{\prime}\left(s_{k, i, 0} \otimes x_{0}^{m-i}\right)
$$

and differentials as

$$
\phi_{1}^{\prime}\left(s_{k, i, 0} \otimes 1\right)=s_{k, i, 0} \otimes x_{0}-f_{i, 0}^{\prime}\left(s_{k, i, 0} \otimes 1\right),
$$

and, for $j \geq 1$,

$$
\phi_{j}^{\prime}\left(s_{k, i, j} \otimes 1\right)=\phi_{i, j}^{\prime}\left(s_{k, i, j} \otimes 1\right)
$$

and

$$
\phi_{j+1}^{\prime}\left(s_{k, i, j} \otimes 1\right)=s_{k, i, j} \otimes x_{0}-f_{i, j}^{\prime}\left(s_{k, i, j} \otimes 1\right)-\phi_{i, j}^{\prime}\left(s_{k, i, j} \otimes 1\right) .
$$

Note the ambiguity of whether an element $s_{k, i, j-1} \otimes 1$ lies in $F_{j}^{\prime}$ or $F_{j-1}^{\prime}$. We will resolve this ambiguity either by an explicit declaration, such as $s_{k, i, j-1} \otimes 1 \in F_{j}^{\prime}$, or implicitly, as in $\phi_{j}^{\prime}\left(s_{k, i, j-1} \otimes 1\right.$ ) (keeping in mind that the differential $\phi_{j}^{\prime}$ is a mapping defined on $\left.F_{j}^{\prime}\right)$.

The modules $F_{j}^{\prime}$ and maps $\phi_{j}^{\prime}$ can also be described in terms of mapping cones. In fact, we have the mapping of complexes $f_{i+1}^{\prime}: F_{i+1, \bullet}^{\prime}(-1) \rightarrow F_{i, \bullet}^{\prime}$, where for each $j$ the map $F_{i+1, j}^{\prime}(-1) \rightarrow F_{i, j}^{\prime}$ is given by $f_{i+1, j}^{\prime}$. Thus this mapping of complexes is ultimately induced by the inclusion $Z_{i} \subset Z_{i+1}$. We also have the mapping of complexes $\mu_{i}: F_{i, \bullet}^{\prime}(-1) \rightarrow F_{i, \bullet}^{\prime}$, where $\mu_{i}(a)=x_{0} a$ is given by multiplying by $x_{0}$. Then, for $j \geq 0$, the modules $F_{j}^{\prime}$ and maps $\phi_{j+1}^{\prime}$ can be seen as coming from amalgamating the mapping cones of $\mu_{m}, f_{m}^{\prime}, \cdots, \mu_{1}, f_{1}^{\prime}$, as shown in Figure 1. Any two consecutive rows give a mapping cone, either for some $\mu$ or for some $f^{\prime}$. The direct sum of the modules in column $j$ gives $F_{j}^{\prime}$. The differential $\phi_{j}^{\prime}: F_{j}^{\prime} \rightarrow F_{j-1}^{\prime}$ is the direct sum of the maps between columns.

Lemma 2.3. The differentials $\phi_{j}^{\prime}, j>0$, form a complex and $\phi_{0}^{\prime} \phi_{1}^{\prime}=0$; i.e., $\phi_{j}^{\prime} \phi_{j+1}^{\prime}=0$ for all $j \geq 0$.

Proof. We have

$$
\phi_{0}^{\prime} \phi_{1}^{\prime}\left(s_{k, i, 1} \otimes 1\right)=\phi_{0}^{\prime}\left(\phi_{i, 1}\left(s_{k, i, 1}\right) \otimes 1\right)=\phi_{i, 0}\left(\phi_{i, 1}\left(s_{k, i, 1}\right)\right) \otimes x_{0}^{m-i},
$$

which equals 0 since $\phi_{i, j} \phi_{i, j+1}=0$, and we have

$$
\begin{aligned}
\phi_{0}^{\prime} \phi_{1}^{\prime}\left(s_{k, i, 0} \otimes 1\right) & =\phi_{0}^{\prime}\left(s_{k, i, 0} \otimes x_{0}-f_{i, 0}\left(s_{k, i, 0}\right) \otimes 1\right) \\
& =\phi_{i, 0}\left(s_{k, i, 0}\right) \otimes x_{0}^{m-i+1}-\phi_{i-1,0}\left(f_{i, 0}\left(s_{k, i, 0}\right)\right) \otimes x_{0}^{m-i+1},
\end{aligned}
$$

which equals 0 since $\phi_{i, 0}=\phi_{i-1,0} f_{i, 0}$. Similarly, for $j>0$, we have

$$
\phi_{j}^{\prime} \phi_{j+1}^{\prime}\left(s_{k, i, j+1} \otimes 1\right)=\phi_{j}^{\prime}\left(\phi_{i, j+1}\left(s_{k, i, j+1}\right) \otimes 1\right)=\phi_{i, j}\left(\phi_{i, j+1}\left(s_{k, i, j+1}\right)\right) \otimes 1=0
$$

and

$$
\begin{gathered}
\phi_{j}^{\prime} \phi_{j+1}^{\prime}\left(s_{k, i, j} \otimes 1\right)=\phi_{j}^{\prime}\left(s_{k, i, j} \otimes x_{0}-f_{i, j}\left(s_{k, i, j}\right) \otimes 1-\phi_{i, j}\left(s_{k, i, j}\right) \otimes 1\right) \\
=\phi_{i, j}\left(s_{k, i, j}\right) \otimes x_{0}-\phi_{i-1, j}\left(f_{i, j}\left(s_{k, i, j}\right)\right) \otimes 1 \\
-\left(\phi_{i, j}\left(s_{k, i, j}\right) \otimes x_{0}-f_{i, j-1}\left(\phi_{i, j}\left(s_{k, i, j}\right)\right) \otimes 1-\phi_{i, j-1} \phi_{i, j}\left(s_{k, i, j}\right) \otimes 1\right),
\end{gathered}
$$

which equals 0 since $\phi_{i-1, j} f_{i, j}=f_{i, j-1} \phi_{i, j}$. 


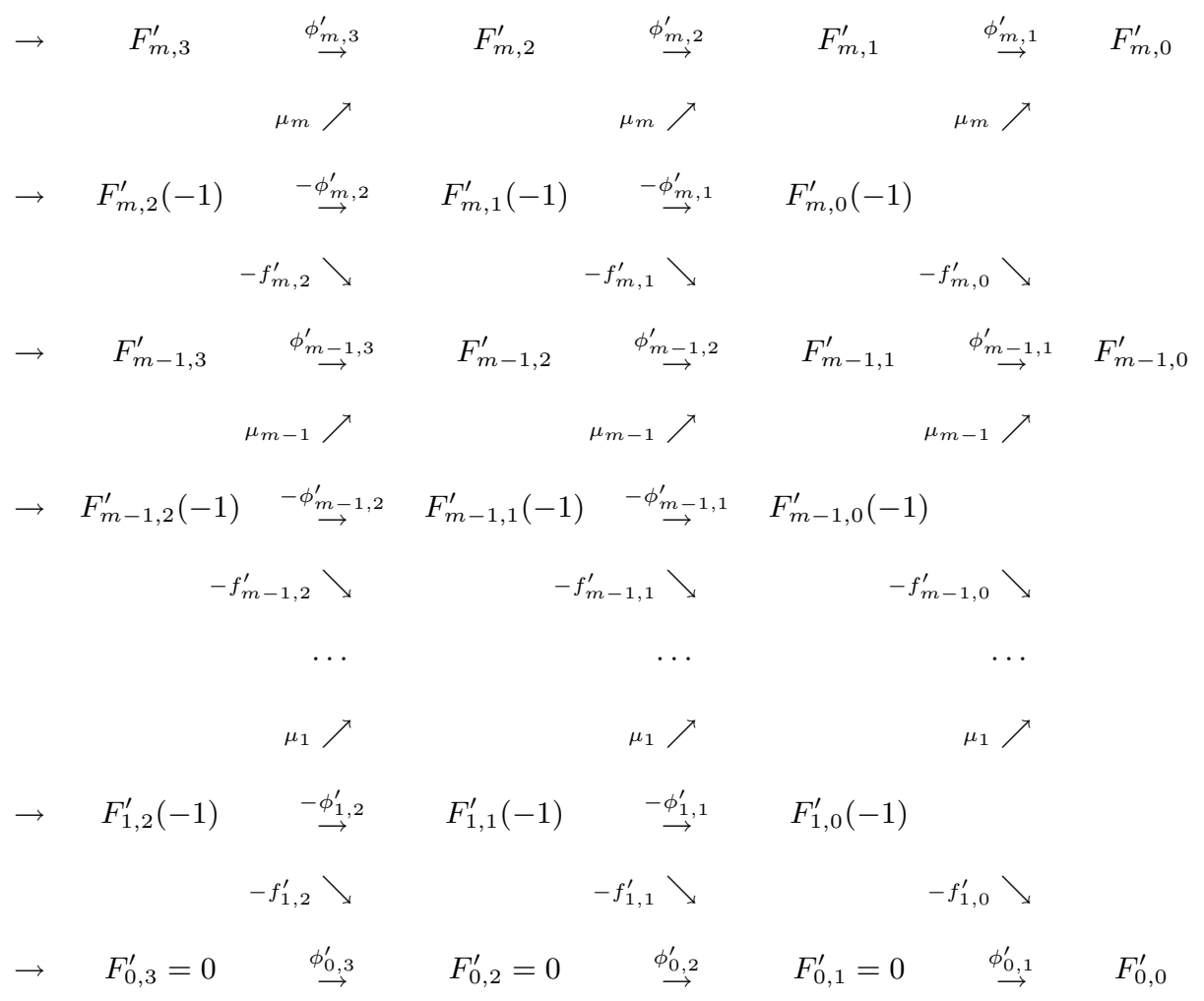

FiguRE 1.

The next result is that this complex gives a resolution. This means first that generators for $I\left(Z^{\prime}\right)$ are given by taking for each $i$ the generators of $I\left(Z_{i}\right)$ times $x_{0}^{m-i}$, and second that syzygies for $I\left(Z^{\prime}\right)$ are of two types. One type comprises the obvious syzygies coming from each of the ideals $I\left(Z_{i}\right)$ individually, which give syzygies of $I\left(Z^{\prime}\right)$ based on the fact that $x_{0}^{m-i} I\left(Z_{i}\right)_{t} \subset I\left(Z^{\prime}\right)_{t+m-i}$. The other type comprises syzygies between elements of $I\left(Z_{i+1}\right)$ and elements of $I\left(Z_{i}\right)$. (These are easy to see too. Not only do we have $x_{0}^{m-i} I\left(Z_{i}\right)_{t} \subset I\left(Z^{\prime}\right)_{t+m-i}$, but given a generator $g$ of $I\left(Z_{i+1}\right)$ of degree $t$, we get an element $a=x_{0}^{m-i-1} g \in I\left(Z^{\prime}\right)_{t+m-i-1}$. Now $g \in I\left(Z_{i+1}\right)_{t} \subset I\left(Z_{i}\right)_{t}$, so we also get an element $b=x_{0}^{m-i} g \in I\left(Z_{i}\right)_{t+m-i} \subset$ $I\left(Z^{\prime}\right)_{t+m-i}$, and of course $x_{0} a=b$, which gives a syzygy of the second type.)

Lemma 2.4. The complex $F_{\bullet}^{\prime}$ gives a resolution of $I\left(Z^{\prime}\right)$.

Proof. We must check that the image of $\phi_{0}^{\prime}$ is $I\left(Z^{\prime}\right)$ and, for all $j \geq 1$, that the image of $\phi_{j}^{\prime}$ is the kernel of $\phi_{j-1}^{\prime}$. Let $f \in I\left(Z^{\prime}\right)$. We may write $f=x_{0} g+h$, where no term of $h$ is divisible by $x_{0}$. By restricting to the hyperplane $x_{0}=0$, we see that $h \in I\left(Z_{m}\right) \subset I\left(Z^{\prime}\right)$, hence that $x_{0} g \in I\left(Z^{\prime}\right)$, and so $g \in I\left(Z_{m-1}^{\prime}\right)$. If $m=1$, then $g \in I\left(Z_{0}^{\prime}\right)=R^{\prime}$, so $f \in x_{0}^{1} I\left(Z_{0}\right)+I\left(Z_{1}\right)$. It follows that $I\left(Z^{\prime}\right)=x_{0} I\left(Z_{0}\right)+I\left(Z_{1}\right)$. If $m>1$, it follows by induction on $m$ that $I\left(Z^{\prime}\right)=x_{0}^{m} I\left(Z_{0}\right)+\cdots+x_{0}^{1} I\left(Z_{m-1}\right)+$ 
$I\left(Z_{m}\right)$, and since the image of $\phi_{i, 0}$ is $I\left(Z_{i}\right)$, it follows from the definition that $\phi_{0}^{\prime}$ maps onto $I\left(Z^{\prime}\right)$, as required.

Now suppose $\phi_{0}^{\prime}(f)=0$. Say $f \in F_{0,0}^{\prime}=R^{\prime}$. Then $\phi_{0}^{\prime}(f)=x_{0}^{m} f$, so $f=0$. Now induct on $i$; say $f \in F_{0,0}^{\prime} \oplus \cdots \oplus F_{i, 0}^{\prime}$. We can write $f=g+a$, where $g \in F_{0,0}^{\prime} \oplus \cdots \oplus F_{i-1,0}^{\prime}$ and $a \in F_{i, 0}^{\prime}$. Write $a=c+x_{0} d$, where $c$ is the sum of the terms of $a$ not involving $x_{0}$. Then $0=\phi_{0}^{\prime}(f)=\phi_{0}^{\prime}(g)+x_{0}^{m-i+1} \phi_{i, 0}^{\prime}(d)+$ $x_{0}^{m-i} \phi_{i, 0}^{\prime}(c)$. If $\phi_{i, 0}^{\prime}(c) \neq 0$, then $x_{0}^{m-i+1}$ divides $\phi_{0}^{\prime}(g)+x_{0}^{m-i+1} \phi_{i, 0}^{\prime}(d)$ but not any term of $x_{0}^{m-i} \phi_{i, 0}^{\prime}(c)$ since $c$ and hence $\phi_{i, 0}^{\prime}(c)$ does not involve $x_{0}$, so we see that $\phi_{0}^{\prime}(g)+x_{0}^{m-i+1} \phi_{i, 0}^{\prime}(d)=0$ and $x_{0}^{m-i} \phi_{i, 0}^{\prime}(c)=0$. Thus, in fact, $\phi_{i, 0}^{\prime}(c)=0$, and hence $c=\phi_{i, 1}^{\prime}\left(c^{\prime}\right)$ for some $c^{\prime} \in F_{i, 1}^{\prime}$. Since we can regard $d$ as being in $F_{i, 0}^{\prime}(-1)$ and $\phi_{1}^{\prime}(d)=x_{0} d-f_{i, 0}^{\prime}(d)$, it is enough now to show $g+f_{i, 0}^{\prime}(d)$ is in the image of $\phi_{1}^{\prime}$. This follows by induction, since $g+f_{i, 0}^{\prime}(d) \in F_{0,0}^{\prime} \oplus \cdots \oplus F_{i-1,0}^{\prime}$ and, by Lemma 2.3. $\phi_{0}^{\prime} \phi_{1}^{\prime}(d)=0$, so $\phi_{0}^{\prime} f_{i, 0}^{\prime}(d)=\phi_{0}^{\prime}\left(\mu_{i}(d)\right)$, hence

$$
\phi_{0}^{\prime}\left(g+f_{i, 0}^{\prime}(d)\right)=\phi_{0}^{\prime}\left(g+\mu_{i}(d)\right)=\phi_{0}^{\prime}(f)=0 .
$$

Next, suppose $\phi_{j}^{\prime}(f)=0$ for some $f$ and some $j \geq 1$. Inducting on $i$, we consider $f \in\left(F_{0, j}^{\prime}\right) \oplus\left(F_{1, j}^{\prime} \oplus F_{1, j-1}^{\prime}(-1)\right) \oplus \cdots \oplus\left(F_{i, j}^{\prime} \oplus F_{i, j-1}^{\prime}(-1)\right)$. The case $i=$ 0 is immediate, so say $i \geq 1$. We can write $f=g+a+b$, where $g \in\left(F_{0, j}^{\prime}\right) \oplus$ $\left(F_{1, j}^{\prime} \oplus F_{1, j-1}^{\prime}(-1)\right) \oplus \cdots \oplus\left(F_{i-1, j}^{\prime} \oplus F_{i-1, j-1}^{\prime}(-1)\right), a \in F_{i, j}^{\prime}$ and $b \in F_{i, j-1}^{\prime}(-1)$. Now $0=\phi_{j}^{\prime}(f)=\phi_{j}^{\prime}(g)-f_{i, j-1}^{\prime}(b)-\phi_{i, j-1}^{\prime}(b)+\phi_{i, j}^{\prime}(a)+x_{0} b$, when $j>1$, and $0=\phi_{1}^{\prime}(f)=\phi_{1}^{\prime}(g)-f_{i, 0}^{\prime}(b)+\phi_{i, 1}^{\prime}(a)+x_{0} b$ for $j=1$. But $\phi_{j}^{\prime}(g)-f_{i, j-1}^{\prime}(b)-$ $\phi_{i, j-1}^{\prime}(b) \in\left(F_{0, j}^{\prime}\right) \oplus\left(F_{1, j}^{\prime} \oplus F_{1, j-1}^{\prime}(-1)\right) \oplus \cdots \oplus\left(F_{i-1, j}^{\prime} \oplus F_{i-1, j-1}^{\prime}(-1)\right)$, for $j>1$, $\phi_{1}^{\prime}(g)-f_{i, 0}^{\prime}(b) \in F_{0,0}^{\prime} \oplus \cdots \oplus F_{i-1,0}^{\prime}$ for $j=1$ and $\phi_{i, j}^{\prime}(a)+x_{0} b \in F_{i, j-1}^{\prime}$, so each is 0 .

Denote by $c$ the sum of all terms of $a$ not divisible by $x_{0}$. Then $a=c+x_{0} d$ for some $d \in F_{i, j}^{\prime}(-1)$. As above we must have $\phi_{i, j}^{\prime}(c)=0$ (hence $c=\phi_{i, j+1}^{\prime}(e)$ for some $\left.e \in F_{i, j+1}^{\prime}\right)$ and $\phi_{j+1}^{\prime}(d)=x_{0} d-f_{i, j}^{\prime}(d)-\phi_{i, j}^{\prime}(d)=x_{0} d-f_{i, j}^{\prime}(d)+b$ (since $\left.x_{0} b=-\phi_{i, j}^{\prime}(a)=-\phi_{i, j}^{\prime}\left(c+x_{0} d\right)=-\phi_{i, j}^{\prime}\left(x_{0} d\right)\right)$, so $\phi_{j+1}^{\prime}(e+d)=a+b-f_{i, j}^{\prime}(d)$. Thus it is enough to show that $f-\phi_{j+1}^{\prime}(e+d)=g+f_{i, j}^{\prime}(d)$ is in the image of $\phi_{j+1}^{\prime}$. But $g+f_{i, j}^{\prime}(d) \in\left(F_{0, j}^{\prime}\right) \oplus\left(F_{1, j}^{\prime} \oplus F_{1, j-1}^{\prime}(-1)\right) \oplus \cdots \oplus\left(F_{i-1, j}^{\prime} \oplus F_{i-1, j-1}^{\prime}(-1)\right)$ and $\phi_{j}^{\prime}\left(g+f_{i, j}^{\prime}(d)\right)=\phi_{j}^{\prime}(g)-f_{i, j-1}^{\prime}(b)=0$, so this follows by induction.

Corollary 2.5. If $I\left(Z_{i+1}\right) \subset R_{1} I\left(Z_{i}\right)$ for each $i$, then the maps $f_{i, j}$ can be chosen so that the resolution $F_{\bullet}^{\prime}$ is minimal.

Proof. By Corollary 2.2, we may assume $f_{i+1, j}: F_{i+1, j} \rightarrow R_{1} F_{i, j}$, for all $i$ and $j$. Since the resolutions $F_{i, \bullet}$ are minimal, we know that the matrix for each map $\phi_{i, j}$ has entries in $R_{1}$, and we now know the same is true for each $f_{i+1, j}$. The same now follows for each $\phi_{j}^{\prime}$ by an inspection of the definition of $\phi_{j}^{\prime}$.

We can now prove Theorem 1.1 .

Proof of Theorem 1.1. Since $F_{0}^{\prime}=\bigoplus_{i=0}^{m} F_{i, 0}^{\prime}$ and $F_{j}^{\prime}=\bigoplus_{i=1}^{m}\left(F_{i, j}^{\prime} \oplus F_{i, j-1}^{\prime}(-1)\right)$ for $j \geq 1$, then, accounting for shifts, we see that the Poincaré polynomial $P\left(Z^{\prime}\right)$ is given by the sum $T^{m}+X T^{m} P\left(Z_{1}\right)+T^{m-1} P\left(Z_{1}\right)+\cdots+X T P\left(Z_{m}\right)+P\left(Z_{m}\right)$, which simplifies to $T^{m}+(1+X T)\left(T^{m-1} P\left(Z_{1}\right)+\cdots+P\left(Z_{m}\right)\right)$, as claimed. 


\section{Applications}

To apply our results to obtain minimal resolutions, we need to verify the condition $I\left(Z_{i+1}\right) \subset R_{1} I\left(Z_{i}\right)$ of Corollary 2.5. We first do this when $\operatorname{char}(K)$ is either 0 or is sufficiently large, then in various additional situations, such as the case of monomial ideals.

Proposition 3.1. Given $p_{i} \in \mathbb{P}^{d}$ and fat points $Z=m_{1}^{\prime} p_{1}+\ldots+m_{r}^{\prime} p_{r}$ and $Y=m_{1} p_{1}+\ldots+m_{r} p_{r}$ with $m_{i}^{\prime}>m_{i}$ for all $i$ whenever $m_{i}^{\prime}>0$, assume char $(K)$ is either 0 or bigger than the degree of each generator in a minimal set of homogeneous generators of $I(Z)$. Then $I(Z) \subset R_{1} I(Y)$.

Proof. This follows easily using Euler's identity, that $\delta F=\sum_{i} x_{i} \partial F / \partial x_{i}$ for any homogeneous form $F$ with either $\operatorname{char}(K)=0$ or $\operatorname{char}(K)>\delta$, where $\delta$ is the degree of $F$.

Proposition 3.2. Let $Y$ and $Z$ be as in Proposition 3.1. Assume that the points $p_{i}$ are located at coordinate vertices of $\mathbb{P}^{d-1}$. Then $I(Z) \subset R_{1} I(Y)$.

Proof. The ideals $I(Y)$ and $I(Z)$ are generated by monomials in this case, and we may assume that the variables are indexed so that $x_{j}$ vanishes at $p_{i}$ for all $i \neq j$. Now assume there is a monomial $f=x_{1}^{n_{1}} \cdots x_{d}^{n_{d}} \in\left(I(Z) \backslash R_{1} I(Y)\right) \subset I(Y)$. Since $f \in I(Z)$, we know $m_{i}^{\prime} \leq\left(n_{1}+\cdots+n_{d}\right)-n_{i}$ for every $i$, but $f \in I(Y) \backslash R_{1} I(Y)$, so $m_{i}=\left(n_{1}+\cdots+n_{d}\right)-n_{i}$ for some $i$. However, $m_{i}^{\prime}>m_{i}$, so this is impossible.

We also have the following bootstrapping result:

Proposition 3.3. Let $p_{1}, \ldots, p_{r} \in L$, where $L \subset \mathbb{P}^{d}$ is a proper linear subspace of $\mathbb{P}^{d}$. Let $R$ be the homogeneous coordinate ring for $L$, and $R^{\prime}$ that for $\mathbb{P}^{d}$. Given positive integers $m_{1}, \ldots, m_{r}$, let $Z=m_{1} p_{1}+\cdots+m_{r} p_{r} \subset L$ be the fat point subscheme of $L$, and let $Z^{\prime}=m_{1} p_{1}+\cdots+m_{r} p_{r} \subset \mathbb{P}^{d}$ be the fat point subscheme of $\mathbb{P}^{d}$ specified by the same multiplicities. If $I\left(Z_{i+1}\right) \subset R_{1} I\left(Z_{i}\right)$ holds for all $i$, then so does $I\left(Z_{i+1}^{\prime}\right) \subset R_{1} I\left(Z_{i}^{\prime}\right)$.

Proof. It is enough by induction to prove this in the case that $L$ is a hyperplane. But by Lemma 2.4 we have that $I\left(Z_{i}^{\prime}\right)=\sum_{0 \leq j \leq i} x_{0}^{i-j} I\left(Z_{j}\right) R^{\prime}$, for all $i$. Thus $I\left(Z_{i+1}^{\prime}\right)=\sum_{0 \leq j \leq i+1} x_{0}^{i+1-j} I\left(Z_{j}\right) R^{\prime}=x_{0}\left(\sum_{0 \leq j \leq i} x_{0}^{i-j} I\left(Z_{j}\right) R^{\prime}\right)+I\left(Z_{i+1}\right) R^{\prime}$, and this is a subset of $x_{0}\left(\sum_{0 \leq j \leq i} x_{0}^{i-j} I\left(Z_{j}\right) R^{\prime}\right)+R_{1}\left(I\left(Z_{i}\right) R^{\prime}\right) \subset R_{1}^{\prime} I\left(Z_{i}^{\prime}\right)$.

Example 3.4. Resolutions of ideals for fat point subschemes supported at up to $d+1$ general points of $\mathbb{P}^{d}$ are known in various cases $([\mathrm{F},[\mathrm{FL},[\mathrm{Fr},[\mathrm{V}])$. Proposition 3.2, and Corollary 2.5 (or Theorem 1.1 for just the Betti numbers), reduce the problem of determining resolutions of fat point subschemes with support at up to $d+1$ general points of $\mathbb{P}^{d}$ to cases in which the support spans the entire projective space. For example, to determine the resolution for $2 p_{1}+2 p_{2}+p_{3}$ for general points $p_{i} \in \mathbb{P}^{d}$ with $d>2$, it is enough to determine the resolutions of $2 p_{1}+2 p_{2}+p_{3} \subset \mathbb{P}^{2}$ and $p_{1}+p_{2} \subset \mathbb{P}^{2}$, and to do $p_{1}+p_{2} \subset \mathbb{P}^{2}$ it suffices to do $p_{1}+p_{2} \subset \mathbb{P}^{1}$. Since, in fact, resolutions for ideals of fat points with support at 3 general points of $\mathbb{P}^{2}$ are known $([\mathrm{C}]$ ), our results as a consequence give the resolution and Betti numbers for ideals of fat points supported at any 3 general points in projective space of any dimension. This generalizes the result for two points $([\mathrm{FL},[\mathrm{V}])$. 
Example 3.5. Another way to generalize the known resolution of fat points with support at two points is to consider supports consisting of collinear points. Let $p_{1}, \ldots, p_{r} \in L$, where $L \subset \mathbb{P}^{d}$ is a line. Let $Z=m_{1} p_{1}+\cdots+m_{r} p_{r} \subset L$. Then $I(Z)=f R$, where $f$ is a polynomial vanishing at each point $p_{i}$ to order $m_{i}$ and $R$ is the homogeneous coordinate ring of $L$. Since the ideals are principal and the degree of the generator is the sum of the multiplicities, it is easy to see that $I\left(Z_{i+1}\right) \subset R_{1} I\left(Z_{i}\right)$ holds for all $i$. Proposition 3.3 and Corollary 2.5 now give the minimal resolution of $I\left(Z^{\prime}\right) \subset \mathbb{P}^{d}$, and Theorem 1.1 gives the Betti numbers, extending the result of $[\mathrm{H}]$ for $d=2$.

Example 3.6. Various facts are known for resolutions of points in $\mathbb{P}^{2}$; our results thus extend these to higher dimension, at least in characteristic 0. For example, C] works out the resolution of the ideal of fat points with support on an irreducible conic in $\mathbb{P}^{2}$, while $[\mathrm{H}]$ determines the Betti numbers for the case of any plane conic, irreducible or not, and $[\mathrm{FHH}]$ determines the Betti numbers for any fat point subscheme with support at up to 8 general points of $\mathbb{P}^{2}$. Thus our results give the resolution for the ideal of fat points whose support lies on an irreducible conic curve in any projective space (since an irreducible degree 2 curve is contained in a plane), and they give the Betti numbers when the support either consists of up to 8 general points in a plane or lies in any conic in a plane, for a plane in any projective space. There are also many additional examples of sets of points $p_{1}, \ldots, p_{r}$ contained in configurations of lines in the plane for which the graded Betti numbers for both $p_{1}+\cdots+p_{r}$ and $2 p_{1}+\cdots+2 p_{r}$ are known (see [GMS]). Our results thus give the graded Betti numbers for these examples regarded as subschemes of $\mathbb{P}^{n}$, by regarding $\mathbb{P}^{2}$ as a linear subspace of $\mathbb{P}^{n}$.

Remark 3.7. We close with a remark about an additional situation in which our criterion for minimality will hold. Consider a fat point subscheme $Z \subset \mathbb{P}^{d}$. For each $i$, let $D_{i}\left(d_{i}\right.$, resp.) be the degree of the generator of maximal (resp., minimal) degree in a minimal set of homogeneous generators for $I\left(Z_{i}\right)$. Since $I\left(Z_{i+1}\right) \subset I\left(Z_{i}\right)$ and $R_{1} I\left(Z_{i}\right)_{t}=I\left(Z_{i}\right)_{t+1}$ for $t \geq D_{i}$, it is clear that the condition $I\left(Z_{i+1}\right) \subset R_{1} I\left(Z_{i}\right)$ holds if the degrees of the generators of $I\left(Z_{i+1}\right)$ are shifted enough with respect to those of $I\left(Z_{i}\right)$ (in particular, if $d_{i+1}>D_{i}$ for each $i>0$ ). This occurs, for example, if, for each $i$, the fat points in $Z_{i+1}$ of multiplicity 1 are general and if there are enough of them. More explicitly, let $Z_{1}$ consist of $r_{0}$ simple points. Let $Z_{2}$ include the same points as does $Z_{1}$, but take these points with multiplicity 2 , and add on $r_{1}$ additional general simple points. Continue in this way, defining $Z_{i}$ and $r_{i}$. The condition $I\left(Z_{i+1}\right) \subset R_{1} I\left(Z_{i}\right)$ holds for all $i$, if, for example, $r_{i} \geq\left(\begin{array}{c}D_{i}+d \\ d\end{array}\right)$ for all $i$, since $I\left(Z_{i+1}\right)$ has no elements of degree less than $D_{i}+1$.

\section{REFERENCES}

[C] M.V. Catalisano, "Fat" points on a conic, Comm. in Alg., 19 (1991), 2153-2168 MR.1123117 (93b:14016)

[F] G. Fatabbi, On the resolution of ideals of fat points, J. Algebra 242 (2001), no. 1, 92-108. MR 1844699 (2002d:13015)

[FL] G. Fatabbi, A. Lorenzini, On the graded resolution of ideals of a few general fat points of $\mathbb{P}^{n}$, J. Pure Appl. Alg. (2005) MR2132878

[Fr] C. A. Francisco, Resolutions of small sets of fat points, J. Pure Appl. Alg. 203 (2005), 220-236 MR2176661

[GMS] A. V. Geramita, J. Migliore, L. Sabourin, On the first infinitesimal neighborhood of a linear configuration of points in $\mathbb{P}^{2}$, preprint (math.AC/0411445 ), 46 pages. 
[H] B. Harbourne, Free Resolutions of Fat Point Ideals on $\mathbb{P}^{2}$, J. Pure Appl. Alg. 125, 213-234 (1998). MR1600024 (99d:13016)

[FHH] S. Fitchett, B. Harbourne and S. Holay, Resolutions of ideals defining eight general fat points of $\mathbb{P}^{2}$, J. Alg. 244 (2001), 684-705. MR1859044 (2002g:14089)

[V] G. Valla, Betti numbers of some monomial ideals, Proc. Amer. Math. Soc., 133 (2005), 57-63. MR2085153(2005f:13013)

Dipartimento di Matematica e Informatica, Università di Perugia, via Vanvitelli 1, 06123 Perugia, Italy

E-mail address: fatabbi@dipmat.unipg.it

Department of Mathematics, University of Nebraska, Lincoln, Nebraska 68588-0130

E-mail address: bharbour@math.unl.edu

Dipartimento di Matematica e Informatica, Università di Perugia, via Vanvitelli 1, 06123 Perugia, Italy

E-mail address: annalor@dipmat.unipg.it 